\title{
Role of XIAP gene overexpressed bone marrow mesenchymal stem cells in the treatment of cerebral injury in rats with cerebral palsy
}

\author{
Wenjing Deng, Chenghe Fan, Yanbo Fang, Yanan Zhao, Yamin Wei, Meng Li and Junfang Teng*
}

\begin{abstract}
Background: This study is performed to investigate the effects of adenovirus-mediated X-linked inhibitor of apoptosis protein (XIAP) overexpressed bone marrow mesenchymal stem cells (BMSCs) on brain injury in rats with cerebral palsy (CP).

Methods: Rat's BMSCs were cultured and identified. The XIAP gene of BMSCs was modified by adenovirus expression vector Ad-XIAP-GFP. The rat model of CP with ischemia and anoxia was established by ligating the left common carotid artery and anoxia for $2 \mathrm{~h}$, and BMSCs were intracerebroventricularly injected to the modeled rats. The mRNA and protein expression of XIAP in brain tissue of rats in each group was detected by RT-qPCR and western blot analysis. The neurobehavioral situation, content of acetylcholine (Ach), activity of acetylcholinesterase (AchE), brain pathological injury, apoptosis of brain nerve cells and the activation of astrocytes in CP rats were determined via a series of assays.
\end{abstract}

Results: Rats with CP exhibited obvious abnormalities, increased Ach content, decreased AchE activity, obvious pathological damage, increased brain nerve cell apoptosis, as well as elevated activation of astrocyte. XIAP overexpressed BMSCs improved the neurobehavioral situation, decreased Ach content and increased AchE activity, attenuated brain pathological injury, inhibited apoptosis of brain nerve cells and the activation of astrocytes in CP rats.

Conclusion: Our study demonstrates that XIAP overexpressed BMSCs can inhibit the apoptosis of brain nerve cells and the activation of astrocytes, increase AchE activity, and inhibit Ach content, so as to lower the CP caused by cerebral ischemia and hypoxia in rats.

Keywords: Cerebral palsy, XIAP, Bone marrow mesenchymal stem cells, Cerebral injury, Astrocytes, AchE

\section{Background}

Cerebral palsy $(\mathrm{CP})$ is defined as a group of permanent movement and posture disorders that results in restricted activity due to non-progressive disturbances that occur early in brain development $[1,2]$. Although it is recognized that various physiological and cognitive symptoms are associated with the disease, chronic pain has been a

\footnotetext{
*Correspondence: tengjunfang0203@163.com
}

The Neurology Intensive Care Unit, The First Affiliated of Zhengzhou University, No.1, Jianshe Road, Zhengzhou 450052, Henan, People's Republic of China subject of clinical and empirical concern in recent years $[3,4]$. The obstruction in diagnosing the syndrome stems from its different classifications, for the reason that $\mathrm{CP}$ can be defined by anatomical brain damage in the cerebral cortex, extrapyramidal system, pyramidal tract, or cerebellum [5]. Routine therapies for CP include orthomorphia, neurotomy, physical therapy, language training, motor function training, and intramuscular injections of botulinum toxin A [6]. Stem cell transplantation is suggested to be a new and promising treatment for $\mathrm{CP}$ [7]. Nevertheless, this procedure is still at the preliminary 
stage of investigation and no clinical trial results have been published so far $[8,9]$.

Several types of stem cells are reported to be candidates for treating $\mathrm{CP}$, including olfactory ensheathing cells, human embryonic neural stem cells, umbilical mesenchymal stem cells (MSCs), as well as bone marrow mesenchymal stem cells (BMSCs) [10, 11]. Autologous MSCs may be a good source of cells, which have been introduced for the treatment of CP due to their roles in tissue repair together with the regulation of immunological processes [12]. In addition, accumulating studies have indicated that human BMSCs show neural phenotypes, which is capable of differentiating into neural stem cell (NSC)-like cells in vitro [13-15]. Based on which, we hypothesized that BMSCs could be used as a novel treatment for the patients with CP. X-linked inhibitor of apoptosis protein (XIAP) is the most effective inhibitor of natural apoptosis through inhibiting the initiator (e.g. caspase-9) and effector (e.g. caspase-3 and -7) caspases along with inhibiting caspase-independent cell death $[16,17]$. It is reported that the regulation of XIAP may be a promising neuroprotective strategy for treating acute and chronic neurodegenerative disorders [18]. Furthermore, a study has shown that combining XIAP with MSC-delivered soluble tumor necrosis factor-related apoptosis inducing ligand (sTRAIL) contributed to the regression of primary tumors without lacking of metastases [19]. Saito et al. have demonstrated that XIAP plays an significant role in the control of the death of apoptotic neuronal cells after transient focal cerebral ischemia [20]. Furthermore, XIAP is of great importance in the apoptosis resistance of HL-60 cells when co-cultured with BMSCs through direct cell contact, and the inhibition of XIAP provides a new strategy for treating acute myeloid leukemia [21]. Therefore, we conducted this study to investigate the effects of adenovirus-mediated XIAP modification of BMSCs on brain injury in rats with $\mathrm{CP}$.

\section{Materials and methods Ethics statement}

The study was approved by the animal ethics committee in The First Affiliated of Zhengzhou University (approval number: 2019-KY-220). All animal work was conducted to relieve their pain according to relevant national and international guidelines.

\section{Experimental animals}

A total of 73 Sprague-Dawley (SD) rats in clear grade (regardless of gender, aging 7 days and weighting $20 \pm 5 \mathrm{~g}$ ) were purchased from Beijing Vital River Laboratory Animal Technology Co., Ltd. (Beijing, China). The rats had free access to eating and drinking in a room with
$12 \mathrm{~h}$ day/night cycle, temperature of $(23-25)^{\circ} \mathrm{C}$, humidity of $45-60 \%$ as well as regular disinfection and ventilation.

\section{Culture and identification of BMSCs in rats}

One rat was euthanized by cervical dislocation, and immersed in $75 \%$ ethanol for $5 \mathrm{~min}$. The tibia and femur were separated in aseptic condition, and the muscle and fibrous tissues were removed. The marrow cavity was washed several times in DMEM/DF12 medium (Gibco, Grand Island, NY, USA) with a $1 \mathrm{~mL}$ injector, until the marrow cavity became white and bright. The cells were suspended and inoculated into a culture flask with the concentration of $1 \times 10^{6}$ cells $/ \mathrm{mL}$ and incubated in an incubator at $37{ }^{\circ} \mathrm{C}$ with $5 \% \mathrm{CO}_{2}$ for 3 days. When the adherent cells reached $80-90 \%$ confluence, the culture medium was removed. The cells were detached with $0.25 \%$ trypsin at room temperature and sub-cultured in the ratio of 1:2. Afterwards, the culture process was repeated every 2 to 3 days, when the cell confluence reached $80-90 \%$, the sub-culture process was repeated.

The cultured and amplified rat's BMSCs at passage 3 were detached with $2.5 \mathrm{~mL}$ trypsin, then washed with PBS containing $10 \mathrm{~g} / \mathrm{L}$ bovine serum albumin (BSA). The cells were suspended for preparing the single cell suspension at a density of $2 \times 10^{5}$ cells $/ \mathrm{mL}$. The cells were added with rabbit anti-mouse CD29, CD44, CD34 and CD4 (each $5 \mu \mathrm{L}$ ) and homotypic control flow antibody and incubated at room temperature in dark for 15-20 min. The cells were detected by a flow cytometer (Becton, Dickinson and Company, Franklin Lake, New Jersey, USA).

When the cells in the culture dish reached $80-90 \%$ confluence, the cells were seeded to the six-well plate, and cultured in the incubator. After reaching $70 \%$ cell confluence, the original culture medium was removed and then cultured in osteogenic differentiation medium $(2 \mathrm{~mL} /$ well, Guangzhou Saiye Biotechnology Co., Ltd., Guangzhou, Guangdong, China). The liquid was changed once every 3 days, five times in total. After abandoning the original culture medium, the cells were fixed with $10 \%$ neutral formaldehyde solution, stained with $1 \mathrm{~mL}$ alizarin red S (Cyagen Biosciences, Santa Clara, CA, USA) for $5 \mathrm{~min}$. The staining was observed under the microscope.

When the cells in the culture dish reached $80-90 \%$ confluence, the cells were inoculated to the six-well plate, and cultured in the incubator. After reaching $100 \%$ cell confluence, the original culture medium was removed and then cultured in adipose induced differentiation medium ( $2 \mathrm{~mL} /$ well, Guangzhou Saiye Biotechnology Co., Ltd., Guangzhou, Guangdong, China). After 3 days, the medium was replaced by growth medium and then changed into adipose induced differentiation medium after $24 \mathrm{~h}$. The above two medium were used alternately, 
with a total of three to five times exchange. The cells were fully cultured for about a week, in which the medium solution was changed every 3 days. After abandoning the original culture medium, the cells were fixed with $10 \%$ neutral formaldehyde solution, stained with $1 \mathrm{~mL}$ Oil red o solution (Cyagen Biosciences, Santa Clara, CA, USA) for $30 \mathrm{~min}$. The staining was observed under the microscope.

\section{BMSCs transfected with adenovirus expression vector Ad-XIAP-green fluorescent protein (GFP)}

BMSCs was inoculated in a 24-well plate at the density of $1.5 \times 10^{5}$ cells/well and cultured for $24 \mathrm{~h}$. The Ad-XIAPGFP virus solution with the multiplicity of infection (MOI) of 100, 200 and 400 were transferred into the BMSCs. After $12 \mathrm{~h}$, the solution was changed and the complete culture medium was added for further culture. The expression of GFP in BMSCs transfected with Ad$\mathrm{XIAP/GFP}$ was observed by a fluorescence microscope to determine the MOI. In order to verify the transfection efficiency, the cells which were infected with the MOI of Ad-XIAP/GFP and Ad-GFP on the 3rd day and the untreated cells were detected by reverse transcription quantitative polymerase chain reaction (RT-qPCR) and western blot analysis to detect the expression of XIAP mRNA and protein in cells. The BMSCs that successfully infected the recombinant adenoviral vector carrying the XIAP gene (Ad-GFP-XIAP) were named XIAP-BMSCs. BMSCs infected with empty vector virus (Ad-GFP) were named NC-BMSCs, and uninfected BMSCs were named as blank. Both Ad-GFP-XIAP and Ad-GFP expression vectors were constructed and identified by Shanghai Sangon Bioengineering Co., Ltd. (Shanghai, China).

\section{Establishment of a rat model of CP}

The rats were numbered according to their body weight and 12 rats were randomly selected as a sham group. The rat model of CP was established by ligating the left common carotid artery and anoxia for $2 \mathrm{~h}$ [22] in the remaining 60 rats. The anesthetized rats were intraperitoneally injected with $1 \%$ pentobarbital sodium for anesthesia. The experimental rats were in supine on the surgical plate, fixed their limbs and heads, and disinfected with iodophor before the neck. The left common carotid artery was found in the triangle area at the junction of the inner side of the sternocleidomastoid and the anterior part of the neck, and the incision was sutured with a No. 0 wire. The experimental rats were immediately placed in an anoxic chamber at $37{ }^{\circ} \mathrm{C}$. The mixture of $8 \%$ oxygen and $92 \%$ nitrogen was continuously filled with the flow rate of $1 \mathrm{~L} / \mathrm{min}$ for $2 \mathrm{~h}$. The whole process of anoxia was continuously monitored by an oxygen meter. In the sham group, the common carotid artery was isolated, and no ligation was performed without hypoxia treatment.

\section{Experimental grouping and treatment}

The successfully-modeled rats were divided into 5 groups, 12 rats in each group: $\mathrm{CP}$ group (establish a rat model of ischemic and anoxic $\mathrm{CP}$ without other treatment), $\mathrm{CP}+\mathrm{PBS}$ group (on the 3rd day after modeling, stereotactic injection of $2 \mu \mathrm{L}$ PBS into the left lateral ventricle of $\mathrm{CP}$ rats), $\mathrm{CP}+\mathrm{BMSC}$ group (on the 3rd day after modeling, stereotactic injection of $2 \mu \mathrm{L}$ BMSCs into the left lateral ventricle of $\mathrm{CP}$ rats), $\mathrm{CP}+\mathrm{NC}$-BMSCs group (on the 3rd day after modeling, stereotactic injection of $2 \mu \mathrm{L} \mathrm{NC-BMSCs}$ into the left lateral ventricle of $\mathrm{CP}$ rats), and $\mathrm{CP}+\mathrm{XIAP}-\mathrm{BMSCs}$ group (on the 3rd day after modeling, stereotactic injection of $2 \mu \mathrm{L}$ XIAPBMSCs into the left lateral ventricle of $\mathrm{CP}$ rats). The rats in the $\mathrm{CP}+\mathrm{PBS}$ group, $\mathrm{CP}+\mathrm{BMSC}$ group, $\mathrm{CP}+\mathrm{NC}$ BMSCs group and CP + XIAP-BMSCs group were fixed on the stereotactic locator on the 3rd day after modeling. The scalp sagittal incision was made under aseptic operation, and $2 \mu \mathrm{L}$ PBS, BMSCs, NC-BMSCs and XIAPBMSCs (about $5 \times 10^{5}$ BMSCs) were injected into the left ventricle via a microinjector.

\section{Evaluation of nerve function injury}

Neurobehavioral assessment of rats was conducted at 3 weeks after modeling.

Morris water maze test: The rats facing the water wall were placed in water several times from 4 water entry points to record the time when they found the hidden platform under the water surface, namely the escape latency.

Suspension test: The rat forelegs grabbed the $0.5 \mathrm{~cm}$ glass rod and observed the time of falling: < $10 \mathrm{~s}$ was 1 point, 10-30 s was 2 points, 31-119 s was 3 points, 2-5 min was 4 points, $>5$ min was 5 points.

Slope test: The rats were placed on a $45^{\circ}$ slope to observe the turning time.

Open field test: The covered box with length and width of $36 \mathrm{~cm}$ was divided into 9 equal size lattices at the bottom of the box, and then to record the activity of rats. More than $1 / 2$ of the body part of the rat entered the adjacent grid was 1 point, and the hind limb of the rat stood was 1 point. The sum of the two points was the total score.

\section{Hematoxylin-eosin (HE) staining}

After the neurological deficit score was finished, 8 rats were taken from each group, and the brain was removed from the neck, and then the brain tissue was quickly separated on the ice. The brain tissue of 4 rats was placed in a prelabeled cryopreservation tube and rapidly put into 
liquid nitrogen, and then transferred to $-80{ }^{\circ} \mathrm{C}$ for the detection of RT-qPCR and western blot analysis. In addition, the brain tissues of 4 rats in each group were fixed in $4 \%$ paraformaldehyde for $24 \mathrm{~h}$, dehydrated, embedded, and sliced with the thickness of $4 \mu \mathrm{m}$, which were used for HE staining, terminal deoxynucleotidyl transferasemediated dUTP nick end-labeling (TUNEL) staining and immunohistochemical staining.

According to the isometric random method, each group of rats was randomly selected with five slices of brain tissue. The slices were dewaxed with xylene for $20 \mathrm{~min}$, and hydrated with $100 \%, 95 \%, 80 \%$ and $70 \%$ for $5 \mathrm{~min}$, respectively, stained with hematoxylin (Beijing Solarbio Science \& Technology Co., Ltd., Beijing, China) for $4 \mathrm{~min}$, differentiated with hydrochloric acid alcohol and washed with running water for $5 \mathrm{~min}$. Next, the slices were stained with eosin (Beijing Solarbio Science \& Technology Co., Ltd., Beijing, China) for $3 \mathrm{~min}$, followed by gradient alcohol dehydration, xylene clearance, and sealing with neutral gum. Tissue staining was observed under an optical microscope (DSX100, Olympus, Tokyo, Japan).

\section{TUNEL staining}

According to the isometric random method, 5 slices of brain tissue were randomly selected from each rat in each group and immersed in $200 \mathrm{~mL} 0.1 \mathrm{~mol} / \mathrm{L}$ sodium citrate buffer solution for $5 \mathrm{~min}$ under microwave heating, and then poured into $80 \mathrm{~mL}$ distilled water immediately. Next, the tissues were added with TUNEL solution ((Boster Biological Technology, Ltd., Wuhan, China) and reacted at $37^{\circ} \mathrm{C}$ for $1 \mathrm{~h}$. After that, the tissues were supplemented with converter-POD and reacted at $37{ }^{\circ} \mathrm{C}$ for $1 \mathrm{~h}$. Lastly, the tissues were counterstained with hematoxylin, followed by gradient alcohol dehydration, xylene clearance, and sealing with neutral gum. The positive cells in the left brain region were counted when the brown granules were stained. Apoptotic index $(\mathrm{AI})=$ the number of positive cells/the total number of cells $\times 100 \%$.

\section{Immunohistochemical staining}

Five slices of brain tissue were randomly selected from each rat in each group and rinsed with PBS for three times, each time for $10 \mathrm{~min}$, fixed with 4\% 1-phosphofructaldolase (PFA) for $10 \mathrm{~min}$, and rinsed with PBS for three times, each time for $5 \mathrm{~min}$. After sealing with 5\% goat serum, the tissues were added with primary antibody, glial fibrillary acidic protein (GFAP) (1:200, Abcam, Cambridge, MA, USA) and incubated at $37{ }^{\circ} \mathrm{C}$ for $2 \mathrm{~h}$. Next, the tissues were rinsed with PBS for three times, each time for $10 \mathrm{~min}$, supplemented with S-P compound and incubated at $37{ }^{\circ} \mathrm{C}$ for $30 \mathrm{~min}$. Afterwards, the tissues were rinsed with PBS for three times, each time for $10 \mathrm{~min}$, supplemented with $0.05 \%$ diaminobenzidine
(DAB) solution, followed by alcohol dehydration, xylene clearance, and sealing with neutral gum. Under the same exposure condition, the images were analyzed by software Image-Pro Plus 6.0 (Media Cybernetics, Rockville, Maryland, USA), and 5 fields of vision were randomly selected to count the average number of positive cells of GFAP.

\section{Transmission electron microscope observation}

The remaining 4 rats in each group were anesthetized by intraperitoneal injection of $1 \%$ pentobarbital sodium after neurobehavioral evaluation. The heart was exposed by opening the chest, and the left ventricle was inserted into the ascending aorta by cutting the left ventricle into the ascending aorta and fixed with hemostatic forceps. The abdominal aorta was clamped with hemostatic forceps. The right atrium was cut open and $50 \mathrm{~mL}$ normal saline containing $12.5 \mathrm{U} / \mathrm{L}$ heparin sodium was quickly infused. After the blood was washed out, 2\% glutaraldehyde was perfused with 100-150 mL glutaraldehyde. About $1 \mathrm{~mm}^{3}$ ischemic brain tissue mass was fixed with $4 \%$ glutaraldehyde for $4 \mathrm{~h}$ and $1 \%$ osmium acid for $2 \mathrm{~h}$, followed by gradient ethanol dehydration, epoxy resin 618 embedding as well as uranium acetate and lead citrate electron staining. The brain cells were observed under a JEM-1400Plus electron microscope (JEOL, Tokyo, Japan).

\section{RT-qPCR}

The Trizol one-step method (Invitrogen, Carlsbad, CA, USA) was implemented to extract the total RNA in brain tissues and BMSCs. The complementary DNA (cDNA) was obtained by avian myeloblastosis virus (AMV) reverse transcriptase after obtaining $1 \mu \mathrm{g}$ RNA. SYBR Green was used for qPCR, and glyceraldehyde phosphate dehydrogenase (GAPDH) was selected as an internal control. PCR primer was designed and synthesized by Invitrogen (Carlsbad, CA, USA) (Table 1). RT-qPCR instrument (ABI 7500, ABI, Foster City, CA, USA) was used for detection. The $2^{-\Delta \Delta \mathrm{Ct}}$ method was used to analyze the ratio relation of target gene expression between the experimental group and the control group. The experiment was repeated in triplicate.

\section{Western blot analysis}

The proteins from brain tissues and BMSCs were extracted and the protein concentrations were determined referring to the instructions of the bicinchoninic acid assay (Boster Biological Technology, Ltd., Wuhan, China). After the proteins were separated by $10 \%$ sodium dodecyl sulfate polyacrylamide gel electrophoresis (Boster Biological Technology, Ltd., Wuhan, China), they 
Table 1 Primer sequence

\begin{tabular}{ll}
\hline Gene & Sequence \\
\hline XIAP & F: 5'-CCCTTGGGAACAGCATGCTA-3' \\
& R: 5'-AATCCAGCACCACAGTAGGC-3' \\
GFAP & F: 5'-AGGCCTAGGCATCTGGAAGA-3' \\
& R: 5'-ATCCTTCTGAGGCCCTCCAT-3' \\
GAPDH & F: 5'-CAGCCGCATCTTCTTGTGC-3' \\
& R: 5'-GGTAACCAGGCGTCCGATA-3'
\end{tabular}

$F$ forward, $R$ reverse, $X I A P$ X-linked inhibitor of apoptosis protein, GFAP glial fibrillary acidic protein, GAPDH glyceraldehyde phosphate dehydrogenase

were transferred to a nitrocellulose membrane using the wet transfer method. Subsequently, the protein samples were transferred to polyvinylidene fluoride membrane and blocked with 5\% BSA for $1 \mathrm{~h}$. Afterwards, the membranes were added with the primary antibodies to XIAP (1: 1000, ab229050), cleaved caspase-3 (1:500, ab49822), Bax (1:1000, ab53154), Bcl-2 (1:1000, ab196495) and $\beta$-actin (1:1000, ab8227). All these antibodies were purchased from (Abcam, Cambridge, MA, USA) and incubated at $4{ }^{\circ} \mathrm{C}$ overnight. The membranes were then rinsed with TBST for 3 times, each time for $10 \mathrm{~min}$. The corresponding IgG secondary antibody labeled by horseradish peroxidase (1: 2000, ab6721, Abcam, Cambridge, MA, USA) were incubated for $2 \mathrm{~h}$ at room temperature so as to wash the membranes for 3 times, each time for $10 \mathrm{~min}$. After DAB coloration, a gel imager was used for photographing (Gel Doc XR, Bio-Rad, Hercules, CA, USA). The gray value analysis of target band was analyzed by Image J software (National Institutes of Health, Bethesda, Maryland, USA).

\section{Enzyme-linked immunosorbent assay (ELISA)}

The brain tissue was thawed at room temperature and weighed it accurately. The content of acetylcholine (Ach) and the activity of acetylcholinesterase (AchE) in the homogenate of rat brain tissue were determined by ELISA kit (Nanjing Jiancheng Institute of Bioengineering, Nanjing, Jiangsu, China) after homogenization in tissue lysate.

\section{Statistical analysis}

All statistical analyses were performed using the SPSS 21.0 software (IBM SPSS, Inc., Chicago, IL, USA). The data were normally distributed by KolmogorovSmirnov test. The measurement data were expressed as mean \pm standard deviation. The $t$ test was used for the comparison between the two groups, and one-way analysis of variance (ANOVA) was used for the comparison among multiple groups. Fisher's least significant difference $t$ test (LSD-t) was used for pairwise comparisons after ANOVA analysis. $P$ values $\leq 0.05$ were considered statistically significant.

\section{Results \\ Morphological observation and identification of BMSCs}

During the initial isolation of rat BMSCs, most of the cells were round in shape, a small number of cells showed irregular shape, and a few blood cells were mixed with them. The cells were suspended in the cell culture medium and varied in size. After $8 \mathrm{~h}$ of culture, some cells were observed to be adhered to the wall, and then to show spindles and strips after $72 \mathrm{~h}$. When the cells were cultured, the cells gradually converged, and the size of the cells increased gradually. After the growth and purification of adhered cells, the proliferation rate of cell culture increased, and the morphology and size of the cells gradually unified, showing a fiber strip (Fig. 1a).

Specific surface markers of isolated cells were identified by flow cytometry. The results showed that surface markers of MSCs, CD29 (98.3\%) and CD44 (97.5\%) [23], were highly expressed while surface markers of hematopoietic stem cells, CD34 (3.7\%) and CD45 (3.1\%) [23], were lowly expressed in cultured rat BMSCs, which were identified as the immunophenotypic characteristics of BMSCs (Fig. 1b).

After differentiation of cultured osteoblasts, a large number of calcium nodules could be seen under an alizarin red staining microscope (Fig. 1c), showing that the cells have good osteogenic differentiation function. After the intervention of the fat-inducing agent, the redstained particles could be seen in the cytoplasm of the cells through the oil-red $\mathrm{O}$ staining, and a large amount of lipid droplets were visible (Fig. 1d), suggesting the existence of adipose differentiation in cells. These results suggest that rat BMSCs were successfully cultured.

\section{Infection efficiency of adenovirus expression vector Ad-XIAP-GFP}

Under an inverted phase contrast microscope, the MSCs infected by the virus was spindle-shaped, with a large nucleus, oval or round, similar to that of uninfected BMSCs. Under a fluorescence microscope, the BMSCs infected by the virus showed green fluorescence in both nucleus and cytoplasm, and the fluorescence intensity was uneven. A small amount of green fluorescence appeared 24 ho after transfection, and the fluorescence increased obviously at $72 \mathrm{~h}$. The infection efficiency was over $90 \%$, and the best transfection efficiency was $\mathrm{MOI}=200$. The infection efficiency of XIAP-BMSCs was similar to that of NC-BMSCs $(92.04 \%$ vs. $90.47 \%)$ (Fig. 2a). 


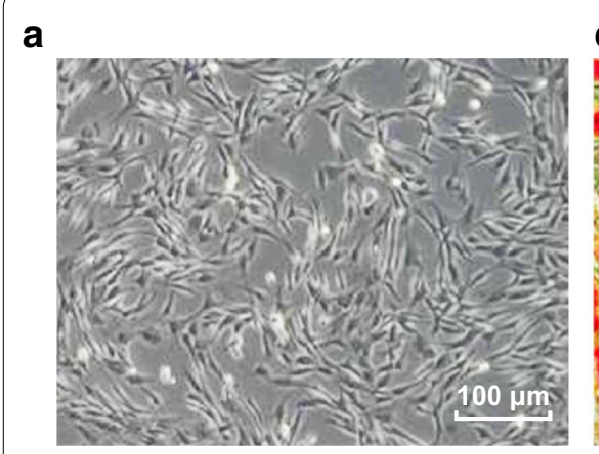

C

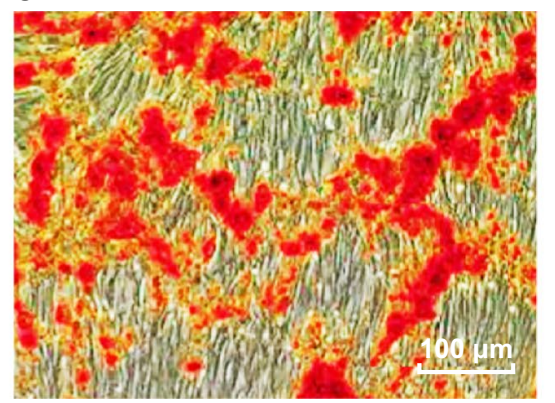

d

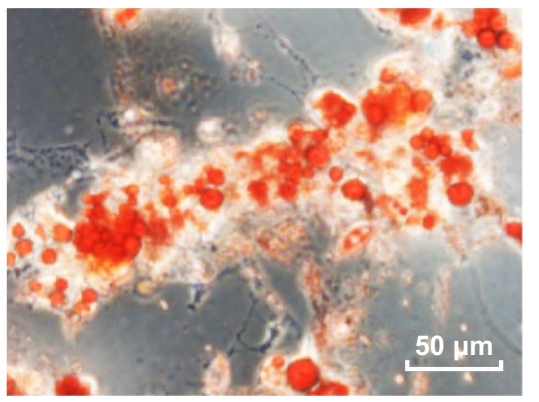

CD44

CD34

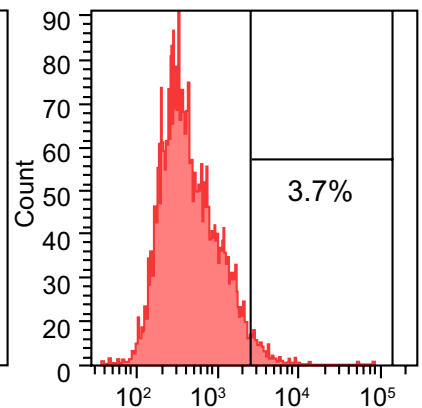

CD45
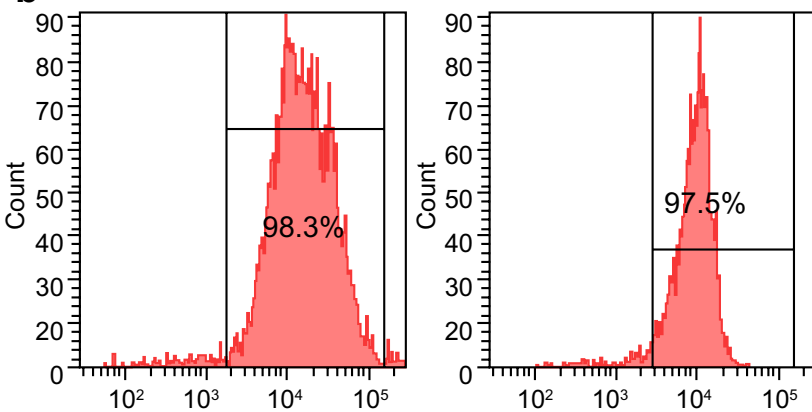

Fig. 1 Morphological observation and identification of rat BMSCs. a The morphology of cells at passage 3 by an inverted microscope $(\times 100)$. $\mathbf{b}$ Identification of cell surface markers by flow cytometry. $\mathbf{c}$ Identification of osteogenic differentiation ability of cells by alizarin red staining ( $\times 100)$. $\mathbf{d}$ Identification of adipogenic differentiation ability of cells by oil red $\mathrm{O}$ staining $(\times 200)$

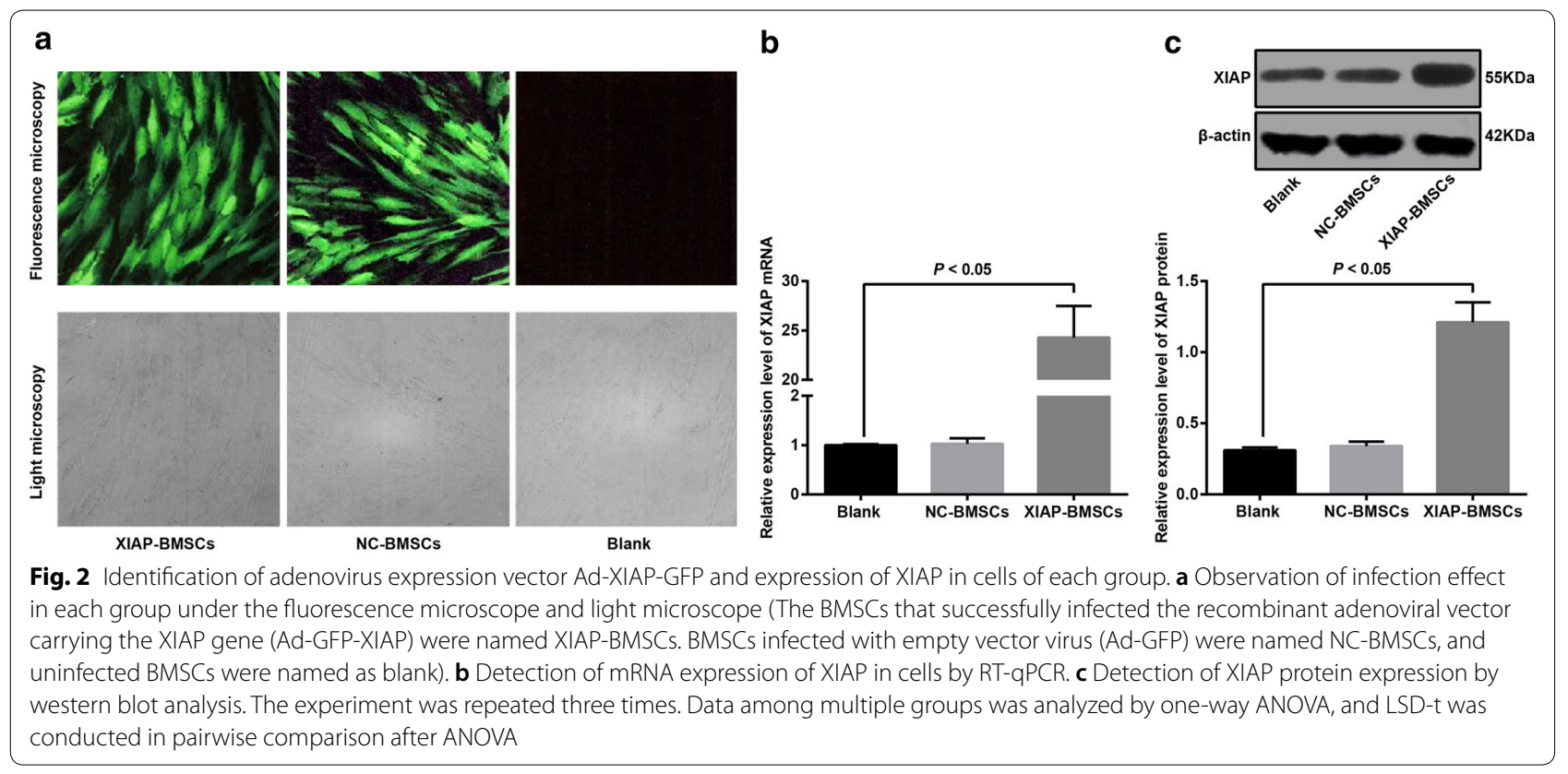


In order to investigate the expression of XIAP after Ad-XIAP-GFP infection with BMSCs, the cells infected with Ad-XIAP-GFP and Ad-GFP on the 3rd day and the untreated cells were selected, and the expression of XIAP mRNA and protein was detected by RT-qPCR and western blot analysis. The results showed that XIAP mRNA and protein expression in NC-BMSCs (BMSCs infected with empty vector virus [Ad-GFP]) had no significant change compared with the blank BMSCs (uninfected BMSCs) $(P>0.05)$. The mRNA and protein expression of XIAP in XIAP-BMSCs (BMSCs that successfully infected the recombinant adenoviral vector carrying the XIAP gene [Ad-GFP-XIAP]) increased significantly compared with the blank BMSCs (uninfected BMSCs) $(P<0.05)$, indicating that XIAP modified BMSCs was successfully constructed (Fig. 2b, c).

\section{XIAP modified BMSCs up-regulated the expression of XIAP in the brain tissues of $C P$ rats}

RT-qPCR and Western blot analysis were used to detect the expression of XIAP in brain tissues of rats in each group. The expression of XIAP mRNA and protein in brain tissues of rats in the $\mathrm{CP}$ group was significantly lower than that in the sham group $(P<0.05)$, suggesting that the expression of XIAP was related to the occurrence of $\mathrm{CP}$. The expression of XIAP mRNA and protein in brain tissues of rats in the CP + XIAP-BMSCs group was significantly higher than that in other $\mathrm{CP}$ groups (all $P<0.05)$. The results showed that XIAP modified BMSCs successfully up-regulated the expression of XIAP in the brain tissues of CP rats (Fig. 3a, b).

\section{BMSCs modified by XIAP improves the neurobehavioral situation in $\mathrm{CP}$ rats}

The experimental results of Morris water maze test indicated that compared with the sham group, the escape latency of rats in the $\mathrm{CP}$ group and the $\mathrm{CP}+\mathrm{PBS}$ group was significantly prolonged (both $P<0.05$ ). Relative to the $\mathrm{CP}$ group and the $\mathrm{CP}+\mathrm{PBS}$ group, the escape latency of rats was significantly shortened in the $\mathrm{CP}+\mathrm{BMSCs}$ group and the $\mathrm{CP}+\mathrm{NC}$-BMSCs group, and that in the $\mathrm{CP}+\mathrm{XIAP}-\mathrm{BMSCs}$ group was further shortened compared with the $\mathrm{CP}+\mathrm{BMSCs}$ group and the $\mathrm{CP}+\mathrm{NC}$ BMSCs group (all $P<0.05$ ) (Fig. 3c).

The results of suspension test suggested that the suspension test score in rats of the CP groups was lower than that in the sham group; among the $\mathrm{CP}$ rats, the $\mathrm{CP}+\mathrm{XIAP}-\mathrm{BMSC}$ group had the lowest score of suspension test, followed by the $\mathrm{CP}+\mathrm{BMSCs}$ group and the $\mathrm{CP}+\mathrm{NC}-\mathrm{BMSC}$ group, which were significantly lower than those in the $\mathrm{CP}$ group and $\mathrm{CP}+\mathrm{PBS}$ group (all $P<0.05$ ) (Fig. 3d).
The results of slope test indicated that after the establishment of CP model, the slope test time of rats was obviously prolonged $(P<0.05)$. After transplantation of BMSCs in CP rats, the slope test time of rats was significantly shortened, while that in rats after transplantation of BMSCs expressing XIAP was further shortened (all $P<0.05$ ) (Fig. 3e).

The results of open field test showed that the open field score in the $\mathrm{CP}$ group and $\mathrm{CP}+\mathrm{PBS}$ group was lower than that in the sham group. After transplantation of BMSCs, the scores of open field test were significantly decreased in rats, and those in the CP+XIAP-BMSCs group were significantly lower than those in the $\mathrm{CP}+\mathrm{BMSC}$ group and the $\mathrm{CP}+\mathrm{NC}-\mathrm{BMSC}$ group (all $P<0.05$ ) (Fig. 3f).

\section{BMSCs modified by XIAP decreases content of Ach and increases activity of AchE in CP rats}

The content of Ach and the activity of AchE in brain tissues of rats were determined by ELISA to further evaluate the brain nerve injury in rats. Compared with the sham group, increased content of Ach and decreased activity of AchE were found in the brain tissues of rats in the $\mathrm{CP}$ and $\mathrm{CP}+\mathrm{PBS}$ groups (both $P<0.05$ ). Relative to the $\mathrm{CP}$ group, the content of Ach was decreased and the activity of AchE was increased in brain tissues of rats in the $\mathrm{CP}+\mathrm{NC}-\mathrm{BMSCs}$ group and the $\mathrm{CP}+\mathrm{XIAP}-\mathrm{BMSCs}$ group (all $P<0.05$ ). In contrast to the $\mathrm{CP}+\mathrm{BMSCs}$ group, the content of Ach was further decreased and the activity of AchE was further increased in brain tissues of rats in the CP + XIAP-BMSCs group (both $P<0.05$ ) (Fig. 4).

\section{BMSCs modified with XIAP attenuates brain pathological injury in CP rats}

The pathological morphology of brain tissues in rats was observed by HE staining. The results of which suggested that in rats of the sham group, brain tissue structure was normal, interstitial edema was not observed, cytoplasm was abundant and nucleus were clearly visible. In rats of the $\mathrm{CP}$ group and the $\mathrm{CP}+\mathrm{PBS}$ group, the brain tissue structure was destroyed, white matter was disordered, which was presented with multiple irregular cystic cavities, cell swelling, degeneration, disordered arrangement, inflammatory cell infiltration and local round softening foci. In rats in the $\mathrm{CP}+\mathrm{BMSCs}$ group and the $\mathrm{CP}+\mathrm{NC}$ BMSCs group, the degree of cell swelling was reduced, inflammatory infiltrating cells were decreased, and cystic degeneration was decreased. In rats of the $\mathrm{CP}+\mathrm{XIAP}$ BMSCs group, the pathological degree of brain tissue was further alleviated and the cells were arranged neatly (Fig. 5a).

The ultrastructure of brain tissues of rats was observed by a transmission electron microscope. In the 


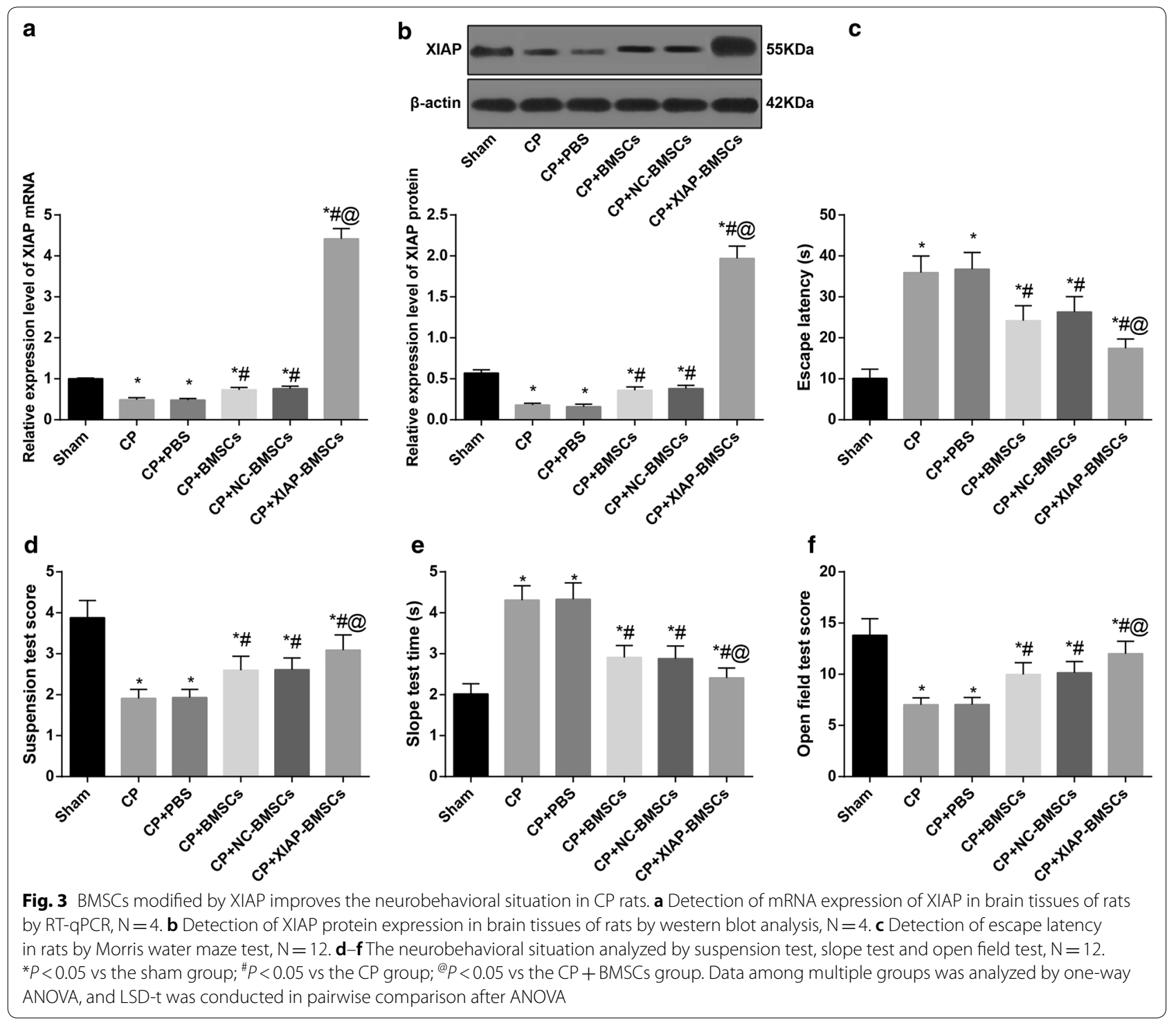

sham group, the neuronal cells of the brain were intact, with abundant rough endoplasmic reticulum, free ribosome and mitochondria, clear mitochondrial ridge, regular arrangement, clear nuclear membrane, dominant chromatin in nucleus and obvious nucleolus. In rats of the $\mathrm{CP}$ group and the $\mathrm{CP}+\mathrm{PBS}$ group, neuronal edema was observed, the number of organelles decreased, most of mitochondria crest and membrane fused or disappeared, some mitochondria became empty, rough endoplasmic reticulum expanded obviously, and degranulation was obvious. Free ribosome reduced, partial double layer nuclear membrane was fused and blurred. In the $\mathrm{CP}+\mathrm{BMSC}$ group and the $\mathrm{CP}+\mathrm{NC}$ BMSCs group, the neurons were slightly edema, the nuclear membrane was intact, only a small amount of shrinkage was found, the nucleus was slightly pyknotic, the chromatin was evenly distributed in the nucleus, and the cytoplasmic organelles were normal. In the $\mathrm{CP}+\mathrm{XIAP}-\mathrm{BMSCs}$ group, the edema state of neurons disappeared, the number of organelles increased significantly, the mitochondrial cristae were broken down, and the vacuolar changes were obviously improved (Fig. 5b).

\section{XIAP modified BMSCs inhibits apoptosis of brain nerve cells in CP rats}

TUNEL staining was used to detect the apoptosis of brain nerve cells in rats. The results showed that there were fewer apoptotic cells in brain tissue of rats in the sham group. The AI of the brain nerve cells in the $\mathrm{CP}$ and the $\mathrm{CP}+\mathrm{PBS}$ groups was significantly higher 

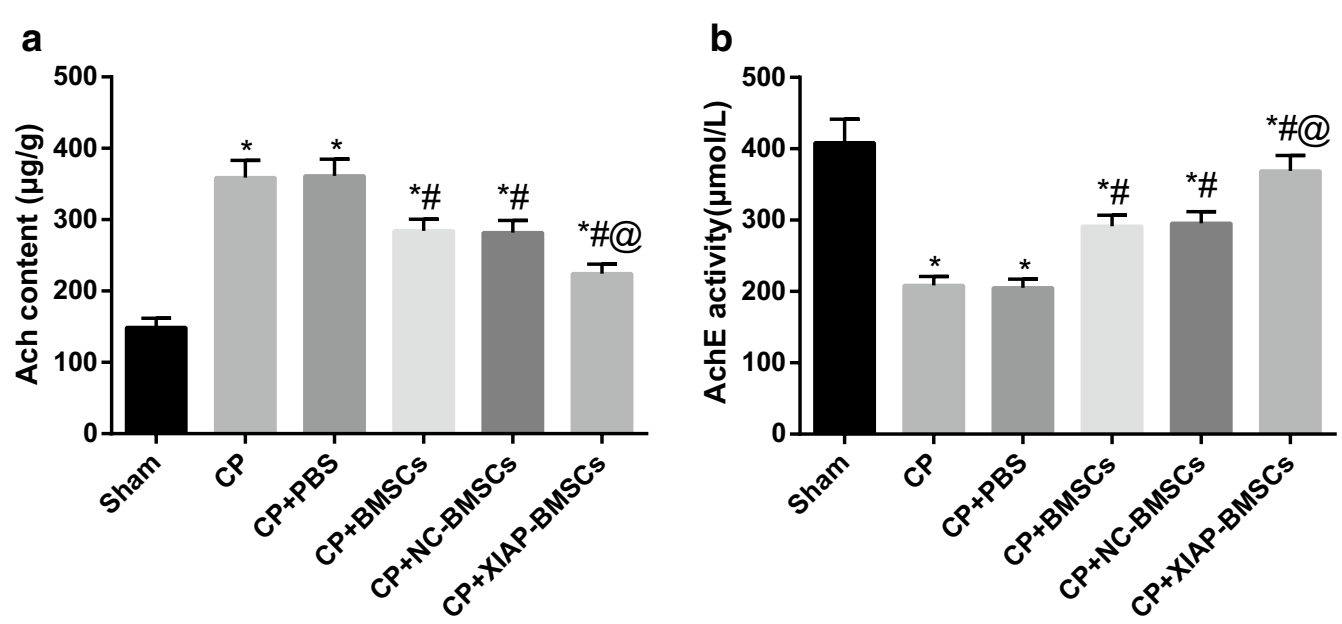

Fig. 4 BMSCS modified by XIAP decreases content of Ach and increases activity of AchE in CP rats. a Detection of Ach content in brain tissues of rats by ELISA, $N=4$. $\mathbf{b}$ Detection of activity of $A c h E$ in brain tissues of rats by ELISA, $N=4$. ${ }^{*} P<0.05$ vs the sham group; ${ }^{*} P<0.05 \mathrm{vs}$ the $C P$ group; ${ }^{@} P<0.05$ vs the CP + BMSCs group. Data among multiple groups was analyzed by one-way ANOVA, and LSD-t was conducted in pairwise comparison after ANOVA
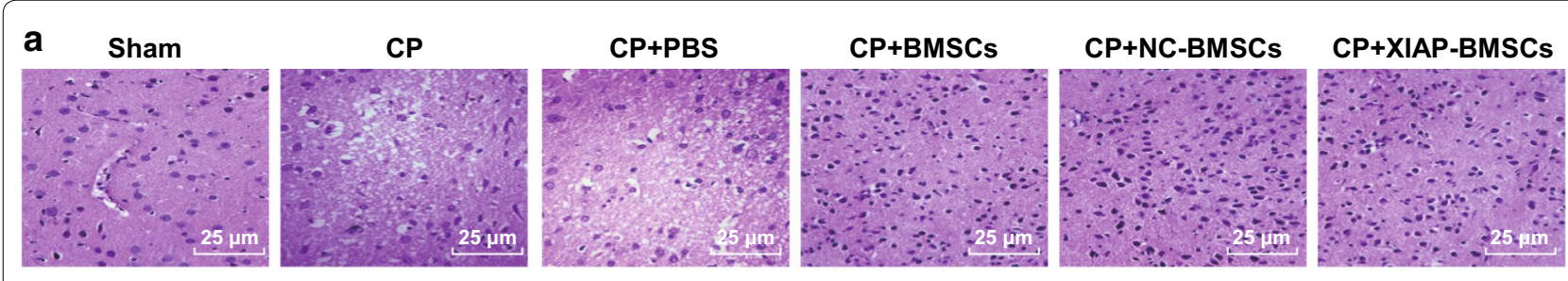

b

Sham

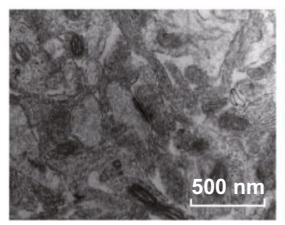

CP

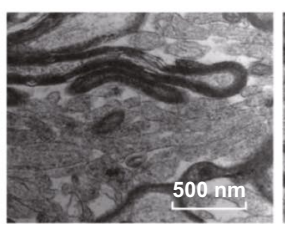

CP+PBS

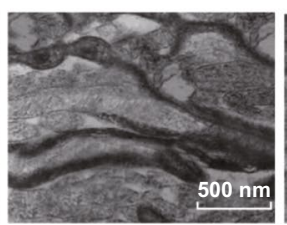

CP+BMSCs

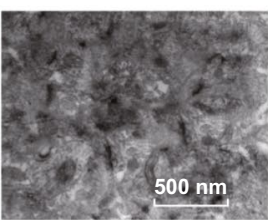

CP+NC-BMSCs

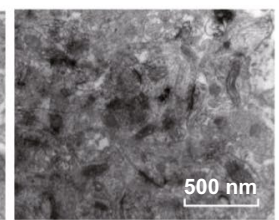

CP+XIAP-BMSCs

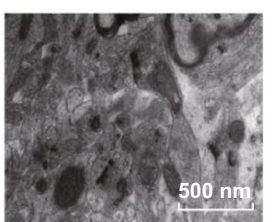

Fig. 5 BMSCs modified with XIAP attenuates brain pathological injury in CP rats. a Pathological observation of brain tissue in rats by HE staining. $\mathbf{b}$ Observation of ultrastructure of rat brain by a transmission electron microscope; $N=4$

than that in the sham group (all $P<0.05$ ). Compared with the $\mathrm{CP}$ group and the $\mathrm{CP}+\mathrm{PBS}$ group, the $\mathrm{AI}$ of brain nerve cells in the $\mathrm{CP}+\mathrm{BMSCs}$ group and the $\mathrm{CP}+\mathrm{NC}$-BMSCs group was significantly decreased (all $P<0.05)$. Relative to the $\mathrm{CP}+\mathrm{BMSCs}$ group and the $\mathrm{CP}+\mathrm{NC}-\mathrm{BMSCs}$ group, the AI of brain nerve cells in the CP + XIAP-BMSCs group was further decreased (all $P<0.05$ ) (Fig. 6a).

Furthermore, the expression of apoptosis-related protein cleaved caspase-3, caspase-3, Bax and Bcl-2 was detected by western blot analysis. The expression of cleaved caspase-3/caspase- 3 was significantly higher while the expression of $\mathrm{Bcl}-2 / \mathrm{Bax}$ was significantly lower in the brain tissues of rats in the CP group than that in the sham group (all $P<0.05$ ). However, the expression of cleaved caspase-3/caspase-3 was significantly lower in the brain tissues of rats in the $\mathrm{CP}+\mathrm{BMSCs}$ group than that in the CP group, and the expression of $\mathrm{Bcl}-2 / \mathrm{Bax}$ was significantly increased (all $P<0.05$ ). The expression of cleaved caspase-3/caspase-3 in brain tissues of rats in the CP + XIAP-BMSCs group was significantly lower and the expression of $\mathrm{Bcl}-2 / \mathrm{Bax}$ was significantly higher than that in the $\mathrm{CP}+\mathrm{BMSC}$ group and the $\mathrm{CP}+\mathrm{NC}$-BMSCs group (all $P<0.05$ ) (Fig. $6 \mathrm{~b}$ ). 


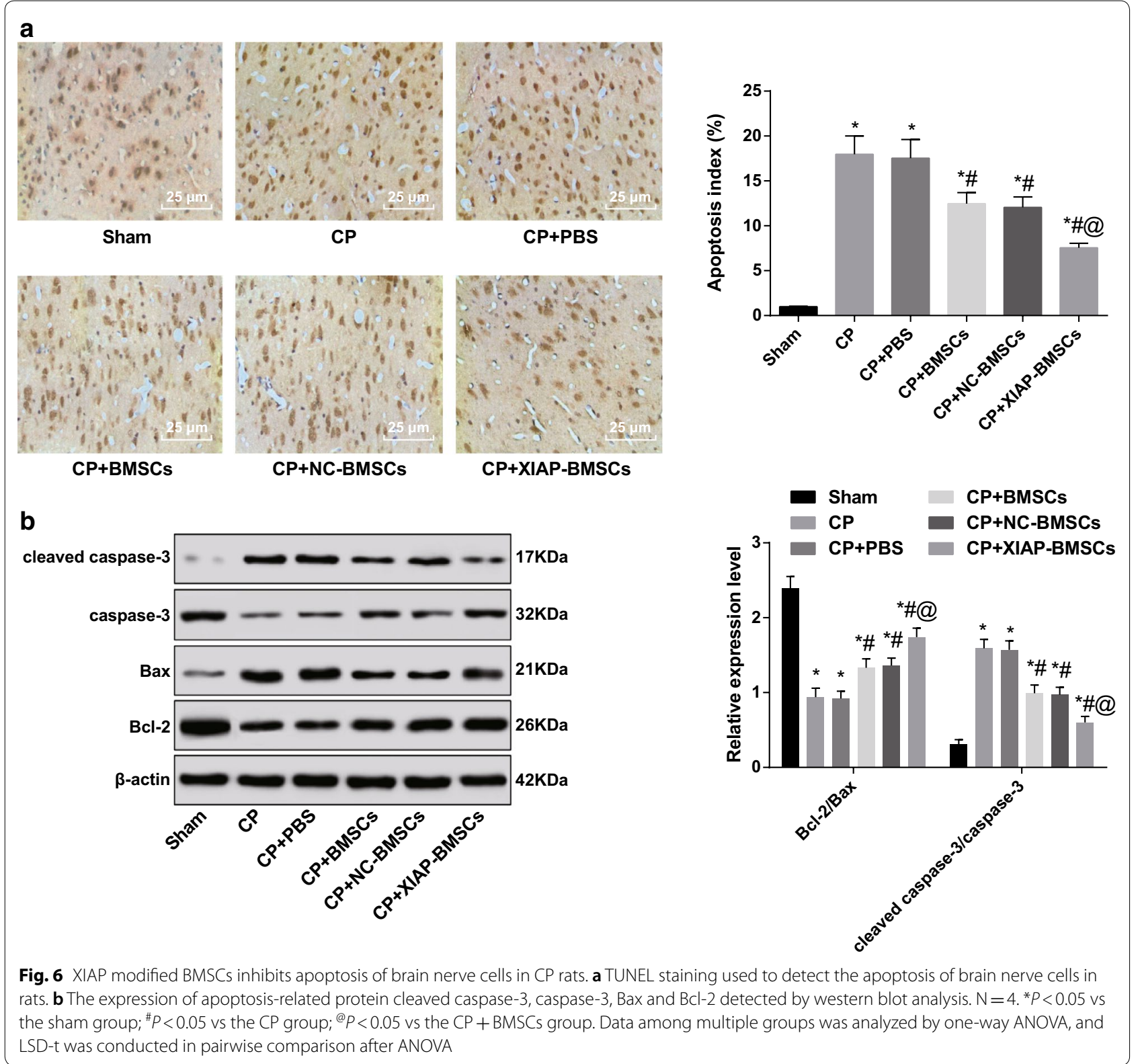

\section{XIAP modified BMSCs inhibits the activation of astrocytes} in $\mathrm{CP}$ rats

RT-qPCR was used to detect the expression of GFAP, a specific marker of astrocytes, in the brain tissues of rats in each group. The expression of GFAP mRNA in brain tissues of rats in the CP group was significantly higher than that in the sham group $(P<0.05)$. There was no significant difference in the expression of GFAP mRNA between the $\mathrm{CP}$ group and the $\mathrm{CP}+\mathrm{PBS}$ group as well as between the $\mathrm{CP}+\mathrm{BMSC}$ group and the $\mathrm{CP}+\mathrm{NC}$ BMSCs group (all $P>0.05$ ). Compared with the CP group, the expression of GFAP mRNA was significantly decreased in the $\mathrm{CP}+\mathrm{BMSC}$ group and the $\mathrm{CP}+\mathrm{NC}$ BMSCs group (both $P<0.05$ ), and that in the CP + XIAPBMSCs group was further decreased compared with that in the $\mathrm{CP}+\mathrm{BMSC}$ group $(P<0.05)$ (Fig. 7a).

GFAP immunohistochemistry was used to detect the positive expression of GFAP in brain tissues of rats, so as to explain the activation of astrocytes. The results showed that the number of GFAP positive cells in brain tissues of rats in the $\mathrm{CP}$ and the $\mathrm{CP}+\mathrm{PBS}$ groups was significantly higher than that in the sham group, while the number of GFAP positive cells in the CP + BMSCs and the $\mathrm{CP}+\mathrm{NC}$-BMSCs group was significantly lower than 

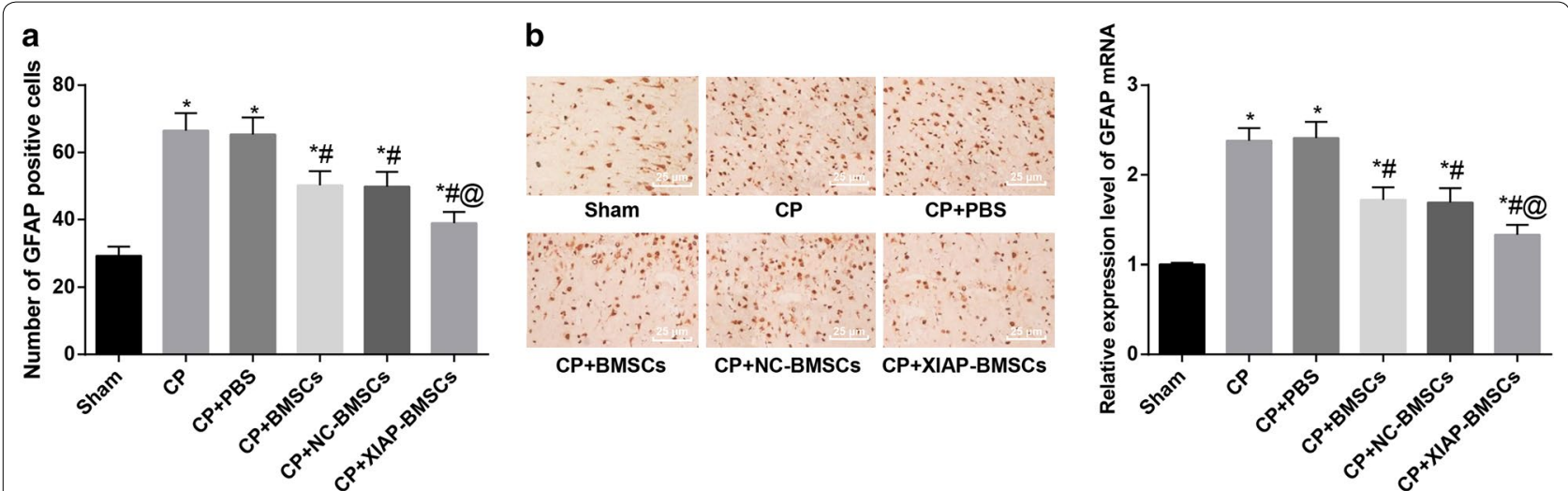

Fig. 7 XIAP modified BMSCs inhibits the activation of astrocytes in CP rats. a RT-qPCR used to detect the expression of GFAP in the brain tissues of rats in each group. $\mathbf{b}$ GFAP immunohistochemistry used to detect the positive expression of GFAP in brain tissues of rats. N $=4$. ${ }^{*} P<0.05$ vs the sham group; ${ }^{\#} P<0.05$ vs the $C P$ group; ${ }^{\circledR} P<0.05$ vs the CP + BMSCs group. Data among multiple groups was analyzed by one-way ANOVA, and LSD-t was conducted in pairwise comparison after ANOVA

that in the CP group (all $P<0.05$ ). The number of GFAP positive cells in brain tissues of rats in the $\mathrm{CP}+\mathrm{XIAP}$ BMSCs group was significantly lower than that in the $\mathrm{CP}+\mathrm{BMSCs}$ group $(P<0.05)$ (Fig. 7b).

\section{Discussion}

The neonatal mortality rate was decreased due to the improvements in perinatal emergency medicine, while the incidence of premature $\mathrm{CP}$ and hypoxic ischemic encephalopathy has increased over time [6]. Routine rehabilitation treatment for $\mathrm{CP}$ is unable to improve the motor function of those patients who with moderate-tosevere chronic CP [24, 25]. Previously, MSCs have been considered as an important experimental tumor therapy based on their intrinsic anticancer properties or integrated with the expression of therapeutic transgenes [19]. In this present study, we aim to investigate the effects of lentivirus-mediated XIAP modification of BMSCs on brain injury in rats with $\mathrm{CP}$. Collectively, the results of this study highlights that BMSCs modified by XIAP can inhibit the apoptosis of brain nerve cells and the activation of astrocytes and increase the activity of AchE, so as to lower the CP caused by cerebral ischemia and hypoxia in rats.

Both cell type and cell modifications play a role in affecting the cells homing after intravascular delivery [26]. The application of BMSCs is particularly promising for the reason that they home to both the injured tissue or secrete factors that induce brain repair [27]. It has been demonstrated that cellular engineering, cell culture conditions as well as cell surface modifications may also regulate cells' migratory behavior and immunomodulatory properties and further improve their therapeutic potential $[28,29]$. Experiments have revealed that transplanted
NSCs show strong plasticity, which is able to integrate with host cells and also become functional substituting nerve cells by establishing a stable synaptic connection [30-32]. At the same time, NSCs may generate neurotrophic factors that promote the recovery of impaired tissues in the damaged brain region [33-35]. As previously reported, MSCs utilized in the model of experimental stroke have elevated the functional recovery of neurological deficits resulted from cerebral ischemia [27]. Besides, another study suggested that MSC transplantation may improve the functional recovery of patients with stroke patients in the absence of adverse side effects [36]. Consistent with these findings, we found that BMSCs is helpful for the treatment of CP to some extent.

In addition, our study also demonstrated that BMSCs modified by XIAP improved the neurobehavioral situation, decreased content of Ach and increased activity of AchE, attenuated brain pathological injury, inhibited apoptosis of brain nerve cells and the activation of astrocytes in CP rats. Several studies have elucidated that in an animal model of cerebral hypoxic-ischemic injury, apoptosis is known as a delayed process of neuronal cell death $[37,38]$. The upregulation of XIAP suppresses ischemic damage in the hippocampus and also recovers neurologic function of adult rat after global ischemia [39]. Wang et al. have stated that the activities of caspase-3 and -9 were virtually abolished; and relative to wild-type mice after hypoxicischemic injury, and XIAP neonatal transgenic mice exhibited decreased tissue loss [40]. In line with the results in our study, neuronal XIAP expression is increased in both developing brain of human and rat after ischemic injury [17]. Meanwhile, overexpression of XIAP in the penumbra of subacute infarcts has been suggested to play a role in the suppression of both 
caspase-dependent and -independent apoptosis in the position of ischemic developing brain [41-43]. Furthermore, it has been revealed that PI3K/Akt/XIAP axis is implicated in the apoptosis resistance of HL-60 cells in the condition of co-culturing with BMSCs through direct cell contact, and the inhibition of PI3K/Akt/XIAP axis may provide a promising therapeutic strategy for the treatment of acute myeloid leukemia [21]. All these aforementioned confirmed the important binding role of XIAP and BMSCs.

XIAP is a major member of the family of endogenous inhibitors of apoptosis (IAPs), which achieves anti-apoptosis by inhibiting different caspases in vitro and in vivo [44]. XIAP is the strongest caspase inhibitor in the IAP family [45]. Ischemic brain injury is a frequent and critical disease of the nervous system. Cerebral ischemia is often accompanied by a large number of neuronal apoptosis, which is closely related to the caspase family. Eberhardt et al. have found that XIAP gene was transfected into rat brain substantia nigra cells by adenovirus in an mitochondrial permeability transition pore-induced animal model of Parkinson's disease. To some extent, upregulation of XIAP can reduce the death of substantia nigra cells and protect dopamine neurons [46]. Simons and Wagenknecht have found that increased expression of XIAP in cerebellar granule cells and glial cells can reduce the damage of such nerve cells. It is speculated that XIAP may have strong anti-apoptotic effect in the central nervous system $[47,48]$. BMSCs not only participate in the repair of neurological function of ischemic brain injury, but also serve as a carrier for gene therapy of cerebral ischemia, while whether overexpression of XIAP in BMSCs can prevent apoptosis in vitro remains to be unearthed, which could be verified in future study if condition permitting (Additional file 1).

\section{Conclusion}

In summary, this present study demonstrates that BMSCs modified by XIAP can alleviate the development of CP caused by cerebral ischemia and hypoxia in rats. However, the mechanism of action underlying BMSCs therapy remains to be fully elucidated, and this persisting gap in our knowledge need further verification.

\section{Supplementary information}

Supplementary information accompanies this paper at https://doi. org/10.1186/s12935-019-0988-6.

Additional file 1. The role of XIAP gene in brain damage of cerebral palsy rats and its molecular mechanism.

\section{Abbreviations}

XIAP: X-linked inhibitor of apoptosis protein; BMSCs: bone marrow mesenchymal stem cells; CP: cerebral palsy; Ach: acetylcholine; AchE: activity of acetylcholinesterase; CP: cerebral palsy; SD: Sprague-Dawley; GFP: green fluorescent protein; RT-qPCR: reverse transcription quantitative polymerase chain reaction; Ad-GFP-XIAP: adenoviral vector carrying the XIAP gene; HE: hematoxylin-eosin; Al: apoptotic index; PFA: phosphofructaldolase; GFAP: glial fibrillary acidic protein; DAB: diaminobenzidine; cDNA: complementary DNA; AMV: avian myeloblastosis virus; GAPDH: glyceraldehyde phosphate dehydrogenase; BCA: bicinchoninic acid; PVDF: polyvinylidene fluoride; BSA: bovine serum albumin; ELISA: enzyme-linked immunosorbent assay; ANOVA: one-way analysis of variance; LSD-t: least significant difference $t$ test.

\section{Acknowledgements}

We would like to acknowledge the reviewers for their helpful comments on this paper.

\section{Authors' contributions}

Guarantor of integrity of the entire study: WD, CF, YF. Study design: WD, CF. Experimental studies: WD, CF, ZZ, YF, YZ, YW, ML. Data analysis: YW, ML, JT. Manuscript editing:YW, ML, JT. All authors read and approved the final manuscript.

\section{Funding}

There are currently no Funding Sources.

Availability of data and materials

Not applicable.

\section{Ethics approval and consent to participate}

The experiment was approved by The First Affiliated Hospital of Zhengzhou University.

\section{Consent for publication}

Not applicable.

\section{Competing interests}

The authors declare that they have no competing interests.

Received: 19 March 2019 Accepted: 1 October 2019

Published online: 23 October 2019

References

1. Van Naarden Braun K, et al. Birth prevalence of cerebral palsy: a population-based study. Pediatrics. 2016. https://doi.org/10.1542/ peds.2015-2872.

2. Rosenbaum P, et al. A report: the definition and classification of cerebral palsy April 2006. Dev Med Child Neurol Suppl. 2007;109:8-14.

3. Hirsh AT, et al. Survey results of pain treatments in adults with cerebral palsy. Am J Phys Med Rehabil. 2011;90(3):207-16.

4. Hirsh AT, et al. Symptom burden in individuals with cerebral palsy. J Rehabil Res Dev. 2010:47(9):863-76.

5. Kulak-Bejda A, et al. Stem cells therapy in cerebral palsy: a systematic review. Brain Dev. 2016:38(8):699-705.

6. Chen $\mathrm{G}$, et al. Neural stem cell-like cells derived from autologous bone mesenchymal stem cells for the treatment of patients with cerebral palsy. J Transl Med. 2013;11:21.

7. Luan Z, et al. Treatment of an infant with severe neonatal hypoxicischemic encephalopathy sequelae with transplantation of human neural stem cells into cerebral ventricle. Zhonghua Er Ke Za Zhi. 2005;43(8):580-3 (discussion 580).

8. Carroll JE, Mays RW. Update on stem cell therapy for cerebral palsy. Expert Opin Biol Ther. 2011;11(4):463-71.

9. Trounson A, et al. Clinical trials for stem cell therapies. BMC Med. 2011;9:52.

10. Li M, et al. Treatment of one case of cerebral palsy combined with posterior visual pathway injury using autologous bone marrow mesenchymal stem cells. J TransI Med. 2012;10:100 
11. Wang $X$, et al. Effects of bone marrow mesenchymal stromal cells on gross motor function measure scores of children with cerebral palsy: a preliminary clinical study. Cytotherapy. 2013;15(12):1549-62.

12. Dan B. Stem cell therapy for cerebral palsy. Dev Med Child Neurol. 2016:58(5):424.

13. Long $X$, et al. Neural cell differentiation in vitro from adult human bone marrow mesenchymal stem cells. Stem Cells Dev. 2005;14(1):65-9.

14. Hermann A, et al. Efficient generation of neural stem cell-like cells from adult human bone marrow stromal cells. J Cell Sci. 2004;117(Pt 19):4411-22.

15. Deng J, et al. Mesenchymal stem cells spontaneously express neural proteins in culture and are neurogenic after transplantation. Stem Cells. 2006;24(4):1054-64.

16. Vaux DL, Silke J. IAPs, RINGs and ubiquitylation. Nat Rev Mol Cell Biol. 2005;6(4):287-97.

17. Askalan $\mathrm{R}$, et al. X-linked inhibitor of apoptosis protein expression after ischemic injury in the human and rat developing brain. Pediatr Res. 2009;65(1):21-6.

18. Zhu C, et al. X chromosome-linked inhibitor of apoptosis protein reduces oxidative stress after cerebral irradiation or hypoxia-ischemia through up-regulation of mitochondrial antioxidants. Eur J Neurosci. 2007;26(12):3402-10

19. Mohr A, et al. Targeting of XIAP combined with systemic mesenchymal stem cell-mediated delivery of sTRAIL ligand inhibits metastatic growth of pancreatic carcinoma cells. Stem Cells. 2010;28(11):2109-20.

20. Saito A, et al. Interaction between XIAP and Smac/DIABLO in the mouse brain after transient focal cerebral ischemia. J Cereb Blood Flow Metab. 2003;23(9):1010-9.

21. Wang $X$, et al. XIAP is upregulated in HL-60 cells cocultured with stromal cells by direct cell contact. Leuk Res. 2007;31(8):1125-9.

22. Aydin A, et al. Erythropoietin exerts neuroprotective effect in neonatal rat model of hypoxic-ischemic brain injury. Brain Dev. 2003;25(7):494-8.

23. Wang B, et al. BMSCs pre-treatment ameliorates inflammation-related tissue destruction in LPS-induced rat DIC model. Cell Death Dis. 2018:9(10):1024.

24. Koman LA, Smith BP, Shilt JS. Cerebral palsy. Lancet 2004;363(9421):1619-31.

25. Holt RL, Mikati MA. Care for child development: basic science rationale and effects of interventions. Pediatr Neurol. 2011;44(4):239-53.

26. Lundberg J, et al. Targeted intra-arterial transplantation of stem cells to the injured CNS is more effective than intravenous administration: engraftment is dependent on cell type and adhesion molecule expression. Cell Transplant. 2012;21(1):333-43.

27. Borlongan $\mathrm{CV}$, et al. The great migration of bone marrow-derived stem cells toward the ischemic brain: therapeutic implications for stroke and other neurological disorders. Prog Neurobiol. 2011;95(2):213-28.

28. Hatlapatka T, et al. Optimization of culture conditions for the expansion of umbilical cord-derived mesenchymal stem or stromal cell-like cells using xeno-free culture conditions. Tissue Eng Part C Methods. 2011;17(4):485-93.

29. Sarkar D, et al. Cell surface engineering of mesenchymal stem cells. Methods Mol Biol. 2011;698:505-23.

30. Park Kl, et al. Neural stem cells may be uniquely suited for combined gene therapy and cell replacement: evidence from engraftment of Neurotrophin-3-expressing stem cells in hypoxic-ischemic brain injury. Exp Neurol. 2006;199(1):179-90.

31. Iwanami A, et al. Transplantation of human neural stem cells for spinal cord injury in primates. J Neurosci Res. 2005;80(2):182-90.
32. Chamberlain $\mathrm{G}$, et al. Concise review: mesenchymal stem cells: their phenotype, differentiation capacity, immunological features, and potential for homing. Stem Cells. 2007;25(11):2739-49.

33. $\mathrm{Ma} \mathrm{H}$, et al. Neural stem cells over-expressing brain-derived neurotrophic factor (BDNF) stimulate synaptic protein expression and promote functional recovery following transplantation in rat model of traumatic brain injury. Neurochem Res. 2012;37(1):69-83.

34. Hofstetter CP, et al. Allodynia limits the usefulness of intraspinal neural stem cell grafts; directed differentiation improves outcome. Nat Neurosci. 2005;8(3):346-53.

35. Lim JY, et al. Neural differentiation of brain-derived neurotrophic factorexpressing human umbilical cord blood-derived mesenchymal stem cells in culture via TrkB-mediated ERK and beta-catenin phosphorylation and following transplantation into the developing brain. Cell Transplant. 2011;20(11-12):1855-66.

36. Bang OY, et al. Autologous mesenchymal stem cell transplantation in stroke patients. Ann Neurol. 2005;57(6):874-82

37. Nakajima W, et al. Apoptosis has a prolonged role in the neurodegeneration after hypoxic ischemia in the newborn rat. J Neurosci. 2000;20(21):7994-8004.

38. Zhu C, et al. Correlation between caspase-3 activation and three different markers of DNA damage in neonatal cerebral hypoxia-ischemia. J Neurochem. 2000;75(2):819-29.

39. Xu D, et al. Attenuation of ischemia-induced cellular and behavioral deficits by X chromosome-linked inhibitor of apoptosis protein overexpression in the rat hippocampus. J Neurosci. 1999;19(12):5026-33.

40. Wang $X$, et al. $X$-linked inhibitor of apoptosis (XIAP) protein protects against caspase activation and tissue loss after neonatal hypoxiaischemia. Neurobiol Dis. 2004;16(1):179-89.

41. Silke J, et al. Direct inhibition of caspase 3 is dispensable for the antiapoptotic activity of XIAP. EMBO J. 2001:20(12):3114-23.

42. Silke J, et al. The anti-apoptotic activity of XIAP is retained upon mutation of both the caspase 3- and caspase 9-interacting sites. J Cell Biol. 2002;157(1):115-24

43. Askalan R, et al. Astrocytic-inducible nitric oxide synthase in the ischemic developing human brain. Pediatr Res. 2006;60(6):687-92.

44. Lotocki G, Keane RW. Inhibitors of apoptosis proteins in injury and disease. IUBMB Life. 2002;54(5):231-40.

45. Deveraux QL, et al. Cleavage of human inhibitor of apoptosis protein XIAP results in fragments with distinct specificities for caspases. EMBO J. 1999;18(19):5242-51.

46. Eberhardt $\mathrm{O}$, et al. Protection by synergistic effects of adenovirusmediated X-chromosome-linked inhibitor of apoptosis and glial cell line-derived neurotrophic factor gene transfer in the 1-methyl-4-phenyl1,2,3,6-tetrahydropyridine model of Parkinson's disease. J Neurosci. 2000;20(24):9126-34

47. Simons M, et al. Adenovirus-mediated gene transfer of inhibitors of apoptosis protein delays apoptosis in cerebellar granule neurons. J Neurochem. 1999:72(1):292-301.

48. Wagenknecht B, et al. Proteasome inhibitor-induced apoptosis of glioma cells involves the processing of multiple caspases and cytochrome $\mathrm{C}$ release. J Neurochem. 2000;75(6):2288-97.

\section{Publisher's Note}

Springer Nature remains neutral with regard to jurisdictional claims in published maps and institutional affiliations. 